$16^{\text {th }}$ International Congress of Metrology, 02008 (2013)

DOI: $10.1051 /$ metrology/201302008

(C) Owned by the authors, published by EDP Sciences, 2013

\title{
The INRIM Water Flow Rate Primary Standard revamping
}

\author{
Carlo Marinari ${ }^{1 \mathrm{a}}$, Fabio Saba $^{2}$ \\ ${ }^{1}$ INRIM - Istituto Nazionale di Ricerca Metrologica, 91 strada della Cacce, 10135 Torino, Italy. \\ ${ }^{2}$ INRIM - Istituto Nazionale di Ricerca Metrologica, 91 strada della Cacce, 10135 Torino, Italy.
}

\begin{abstract}
In 2011, INRIM opened a noteworthy upgrading work to plane an extensive renovation of the national water flow rate primary standard, to get better its structural features and to increasing its metrological performance. The paper presents the main activity of revamping of the INRIM liquid flow calibration rig in order improve its flow rate range and, mainly, to update its ancillary instrumentation towards a more modern approach to signal conditioning, by introducing automatic and user-friendly control of the measurement-taking process.An evaluation of uncertainty propagation in flow rate measure using a Monte Carlo method is adopted.
\end{abstract}

\section{Introduction}

The Italian water flow rate primary standard is a flow calibration rig based on the static weighing gravimetric system with flying start and finish, as established by standard EN-ISO 24185.

Its flow rate capacity ranges from $0.2 \mathrm{~kg} / \mathrm{s}$ to $7 \mathrm{~kg} / \mathrm{s}$. The rig is equipped with four different measurement lines (pipe bore from $25 \mathrm{~mm}$ up to $50 \mathrm{~mm}$ ), a $150 \mathrm{~kg}$ balance and a high speed (less than $4 \mathrm{~ms}$ ) flow diverter. The temperature of tests can vary from $18{ }^{\circ} \mathrm{C}$ up to $80^{\circ} \mathrm{C}$.

The plant includes the devices required to generate and maintain constant flow rate, pressure and temperature. The ratio between accumulated water mass and flow deviation time provides the value of the mass flow:

$$
q_{m}=k \frac{P+C}{\tau}
$$

where:

$\mathrm{P}$ is the mass of fluid indicated by the balance;

is the time of water collection;

$\mathrm{k}$ points out the correction factor for the buoyancy exerted on the fluid, and losses due to evaporation and splashing during filling of the weighing reservoir;

$\mathrm{C}$ indicates the correction to be applied to readings following calibration of the balance and thermal effects on the measurement.

Consequently, the flow rate in volume is:

$$
q_{v}=\frac{q_{m}}{\rho}
$$

where $\rho$ is the density of the fluid at the measurement temperature, expressed in $\mathrm{kg} / \mathrm{m}^{3}$.

\section{General plant setup}

The facility was designed and built in the 1980s, and still is a high-end calibration rig but it, obviously, presented a deep gap in terms of efficiency and reliability of process controls, signal acquisition and computer interface.

Therefore, in 2011, INRIM planned and implemented extensive renovation works to improve the above flow rate standard features.

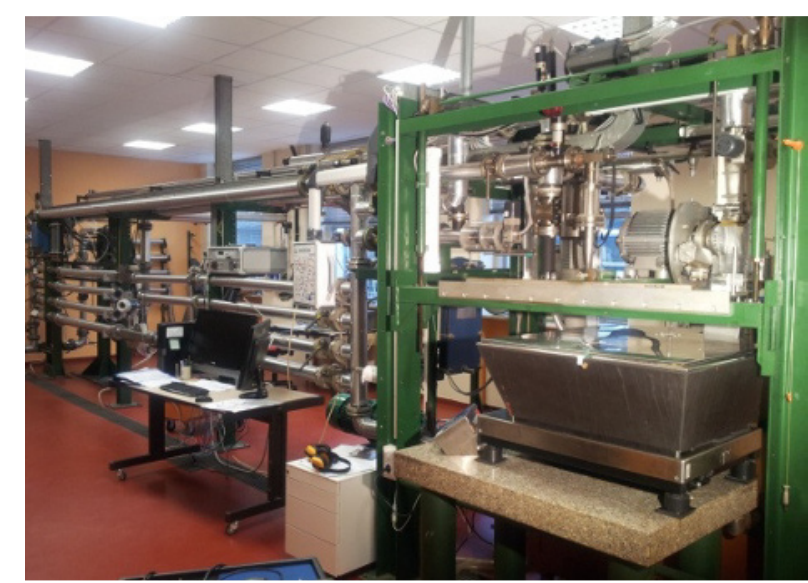

Fig 1 - Overview of the renewed INRIM water flow rate standard.

This activity enabled to improve the test rig (fig. 1) flow rate range (up to $10 \mathrm{~kg} / \mathrm{s}$ ), its metrological characteristics and, mainly, to update its ancillary instrumentation towards a more modern approach to signal conditioning, by introducing automatic and user-friendly control of the measurement-taking process.

Special attention was focused on liquid flow diversion (fig 2).

\footnotetext{
ac.marinari@inrim.it
} 


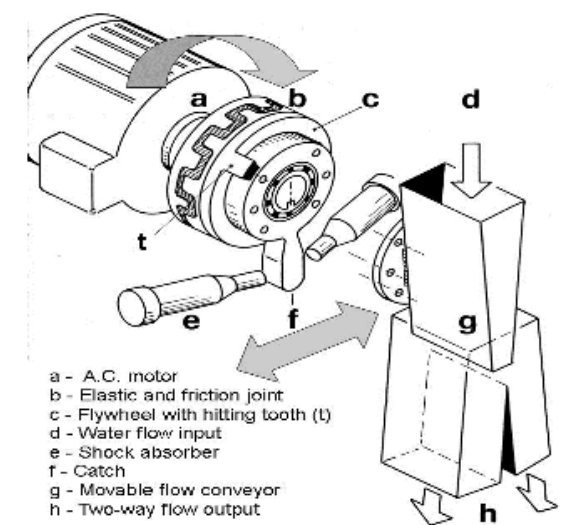

Fig 2. Scheme of the flow diversion device.

This is an essential part of the "flying start and finish" calibration method, as it causes liquid collection in the weighing tank by directing the liquid flow into the tank on initiation of the process and redirects the flow back to the bypass circulation once collection has been completed. In order to correctly measure the collection time, the diverter also provides timer actuation when changing position.

Collection and timer actuation times are the periods between times $t_{0}$ through $t_{3}$, and $t_{\text {start }}$ through $t_{\text {stop }}$, respectively. (fig. 3)

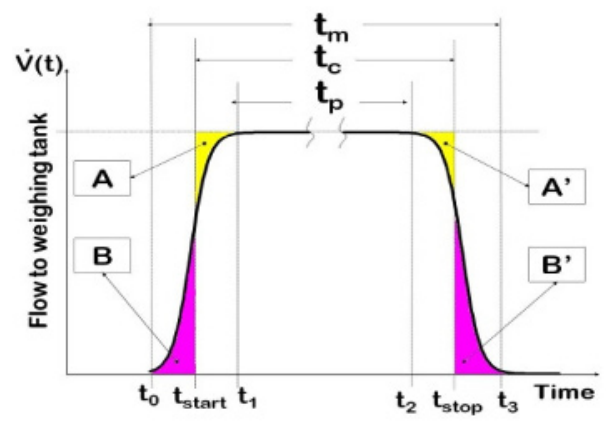

Fig.3. The flow diversion principle

The lateral flow profile along the trajectory, which passes through the diverting edge, is an important quantity of influence. It can be roughly described as a linear function versus the diverter motion path that starts at time $t_{0}$ and ends at $t_{1}$ upon diversion from the bypass to the weighing tank. The second transitional process of diversion occurs during the period of time from $t_{2}$ through $t_{3}$, when the liquid flow is redirected back to the bypass flow condition. The need to minimize potential errors during transitions $t_{0}$ $t_{1}$ and $t_{2}-t_{3}$ has suggested making the deviation time as short as possible. This involves high values of kinetic energy with subsequent considerable mechanical stress on the diverter.
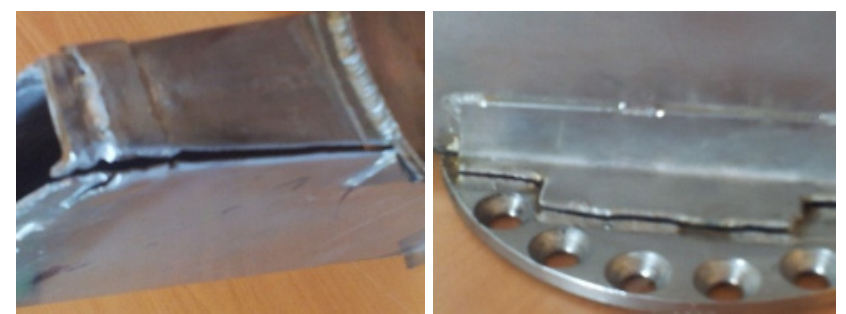

Fig 4: Diverters breakdown.
This structural problem required the shunt system to be redesigned, which was achieved through numerical analysis and tests conducted on various geometrical shapes. (fig 5)

A critical design-related requirement during this process was to avoid weight increases in order not to enhance acceleration stresses and to maintain movement velocity.

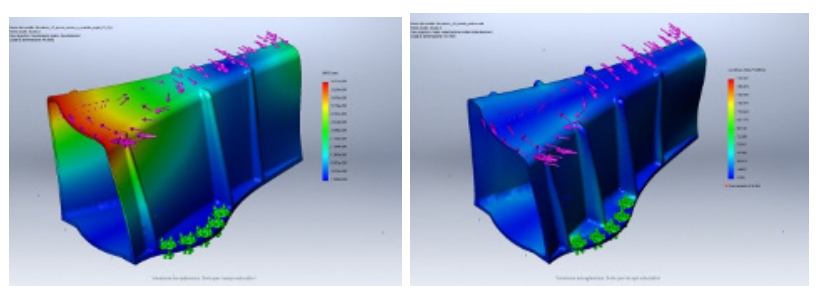

Fig 5: Examples of FEM design of different flow diverter prototypes.

The problem was solved by using the ERGAL7050 alloy $(2.6 \mathrm{Cu} ; 3.1 \mathrm{Mg} ; 6.7 \mathrm{Zn})$, instead of stainless steel. It has a density of $2.7 \mathrm{~g} / \mathrm{cm}^{3}$ versus $7.9 \mathrm{~g} / \mathrm{cm}^{3}$ of steel. Hence a thicker diverter was built (greater elasticity), while maintaining the same total weight.

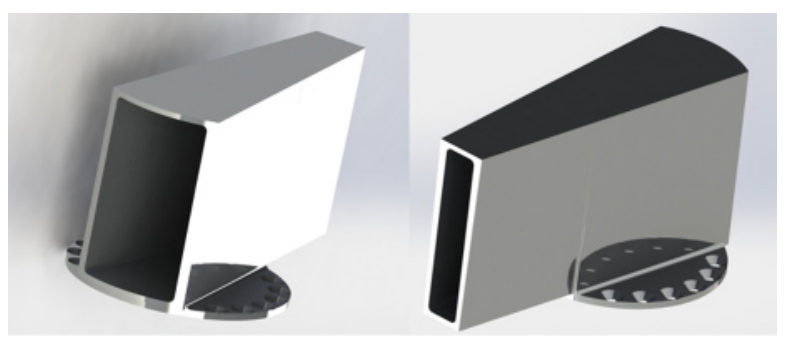

Fig. 6 New diverter

Moreover, the new diverter was built in one piece with laser technology from a $13 \mathrm{~kg}$ of massive ERGAL block. Its final weight is $840 \mathrm{~g}$ (fig.6)

Measurement instruments were also improved to ensure stricter control of all metrological quantities that give a contribute of uncertainty to the measurement, namely flow rate, temperature, pressure, water density, deviation time, balance readout, ambient-air condition and atmospheric pressure.

\section{Human-machine interface and data presentation.}

The calibration of the DUT runs fully automatically, with the capabilities of the computer-based and a

"Supervisory Control And Data Acquisition for Human Machine Interface" system (SCADA/HMI) being utilized for automatic process control and operator interaction. The SCADA/HMI platform Movicon 11.2 (by PROGEA srl) is a realistic interface with efficiency and performances based on emergent and multiplatform technologies, with graphic symbols completely customizable, using VBA scripts as well, and symbols with Power Template technology. 
The operator has full access to all process variables of the calibration plant directly interacting with the two monitors: monitor 1 for process control (fig. 7) and monitor 2 for readout measurement and testing condition, as in fig 8 .

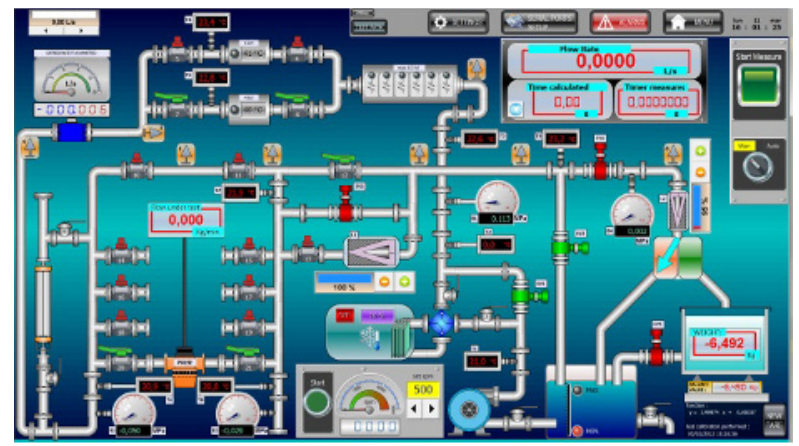

Fig. 7 Control panel layout

The graphic lay out represents realistically the flow rate standard plant, and any graphic symbols are active keys to sensing and actuating devices, their status and the actual measurement values.

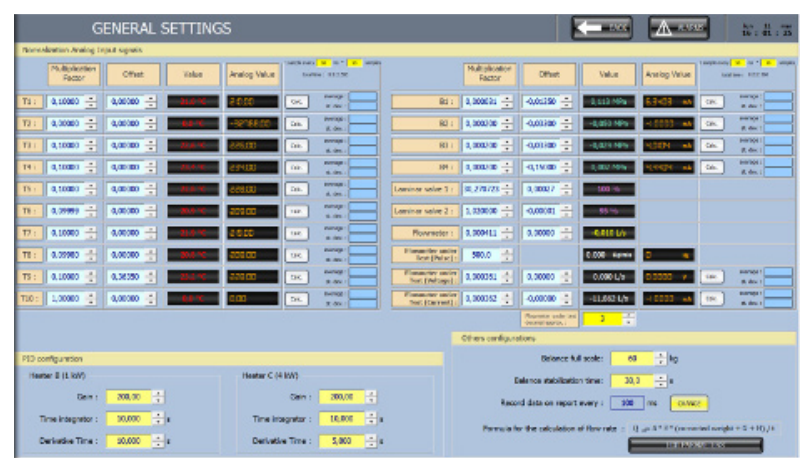

Fig. 8 Monitor 2 with the complete read outs screen

Virtual on-screen buttons are the "active points" to control, by mouse, valves, heaters, cooler, diverter, balance... and to check, in real time, all the measurement readout: flow, pressure, temperature, time

Further windows are achievable to balance calibration, input/output digital port program and testing procedure definitions.

\section{4- Measurement data correction}

In a flow rate standard based on the static weighing gravimetric system with flying start-and-finish the metrological quantities to measure are mass and time of the water collection.

The weighing system and the flow diversion have a direct impact on measurement uncertainty of the measurement process.

\subsection{Weighing system}

The gravimetric-reference system comprises a $150 \mathrm{~kg}$ balance by Toledo Mettler, with resolution of $0.1 \mathrm{~g}$ and nominal expanded uncertainty of $1 \mathrm{~g}$.
It is controlled by the SCADA/HIM too, that sets up a readout delay in order to avoid the influence of the water oscillation into the balance tank on the final weight value. The formula /1/ can also be written as:

$$
q_{m}=\frac{W}{\tau} \quad\left(1_{b i s}\right)
$$

Where $\mathrm{W}$ is the weight of water indicates by the balance and corrected as in formula:

$$
W=A \cdot E \cdot(B+F+G+H)
$$

Where: $\mathrm{A}=$ correction factors for the water buoyancy; $\mathrm{E}=$ correction coefficient for losses due to evaporation and splashing; $\mathrm{B}=$ balance readouts; $\mathrm{F}=$ correction for balance calibration; $\mathrm{G}=$ correction for thermal effect; $\mathrm{H}=$ supplementary water buoyancy factors at high temperature

\subsection{Collection time and diversion}

The flow diverter represents a critical component part of a flow calibrator as it has a direct impact on measurement uncertainty. Thus the supervision of the diverter's reproducible operation and the weighing time are of essential importance.

The flow rate which is directed into the weigh tank is, of course, a function of time. It is zero while the water is flowing through the by-pass into the storage tank and when the diverter is actuated, the transition time $T_{\text {jet }}$ of the diverting edge through the jet starts.

The flow rate into the weigh tank increases up to the value in the test rig. This rise depends mainly on the speed of the diverting edge, the shape of the jet's cross section, the velocity profiles across the jet width and the start/stop time switch.

The process of switching back can be described by analogue considerations.

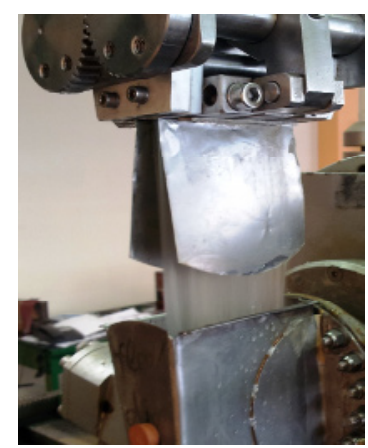

Fig. 9 Rectangular shape of water flow jet.

The diverter was optimized in order to combine the shortest possible diversion time possible with good repeatability capabilities. A rectangular shape of the jet is guaranteed by the laminar valve, as shown in fig 9 .

Another important factor in the measurement process is flow behavior during diversion. It was filmed using a high speed camera to detect any anomalous behavior that could lead to systematic and hidden errors.

This highlighted some asymmetrical features in flow diversion and enabled detection of a spurious reflux of fluid that could slightly alter the final mass value. 
Fig. 10 shows some images of the recording at 1200 frames/s.

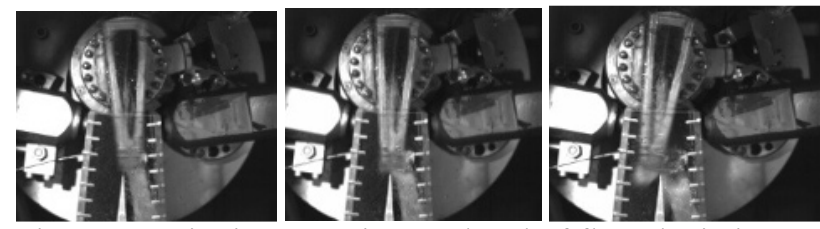

Fig. 10 Beginning, transient and end of flow deviation

The results of these tests guided the design of the new diverter profile (the process was carried out in parallel with structural redesigning) and suggested adopting a seethrough collector in order to achieve direct and real-time monitoring of flow performance during every step of the measurement-taking procedure (fig. 11)

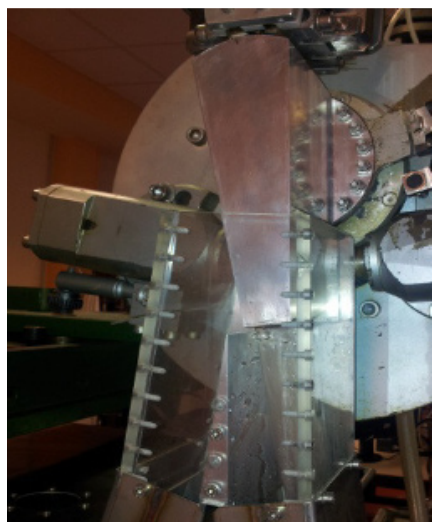

Fig. 11 INRIM see-through collector

\section{Preliminary experimental results}

In the period between September 2012 and February 2013 a reference Coriolis mass flowmeter was used in five European NIMs ( Tubitak UME,Turkey; Lithuanian Energy Institute Central office of Measurement, Poland; INRIM, Italy and Bulgarian Institute of Metrology, Bulgary) to compare calibrations recorded by water flow standard facilities in different laboratories.

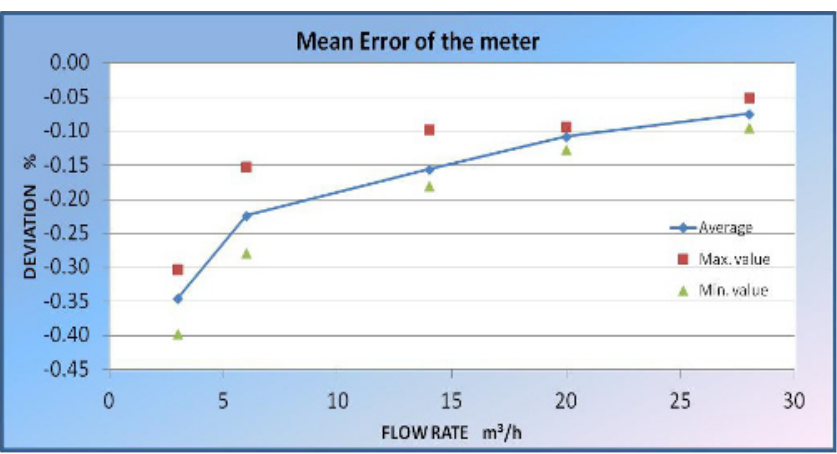

Fig. 12 Mean error of the Euramet project transfer reference meter vs INRIM flow ring.

The comparative study was carried out as part of the EURAMET project $n^{\circ} 1233$ "Inter-comparison of water meter reference standard." The tests were conducted with flow range from $3 \mathrm{~m}^{3} / \mathrm{h}$ to $28 \mathrm{~m}^{3} / \mathrm{h}$, and the main INRIM results are shown in Fig 12.

\section{Evaluation of uncertainty propagation using a Monte Carlo method}

In order to get a more detailed characterization of the flow rate measures, the assessment of the propagation of the probability distributions of uncertainties associated with the various measured and calculated quantities involved in flow rate measure can be performed by the Monte Carlo method.

This method, through the simulation of a statistically significant number of repeated water flow rate measure experiments, allows determining the probability density function associated with the flow rate measure, taking into account how the uncertainties related to the various measures of temperature, pressure, volumetric and gravimetric flow rate, time and to the calculated properties propagate during the measure experiment.

The number of trials can be chosen a priori or can be determined as function of the desired tolerance on the relative standard deviation of the flow rate mean value.

The Monte Carlo analysis of uncertainty propagation in flow rate measurement has been implemented in a Matlab routine in order to preliminary evaluate the related uncertainty as performed by the INRIM water flow rate standard; the models used for flow rate measure calculation is described by equations (1) and (2).

The Matlab routine allows setting all the uncertainties related both to the various measured quantities, as determined by the measuring instruments, and to the correction factors used in water flow rate calculation formulas. It leads towards the estimation of the probability density function of water flow rate. All the measured quantities are considered as non-correlated.

The simulated gravimetric and volumetric flow rate measurements are carried out at the water temperature of $80{ }^{\circ} \mathrm{C}$.

Table 1. Setting of experimental conditions of the various measuring quantities.

\begin{tabular}{cccc}
\hline Quantity & Input Value & $\begin{array}{c}\text { Relative } \\
\text { uncertainty }\end{array}$ & $\begin{array}{c}\text { Probability } \\
\text { Density } \\
\text { Function }\end{array}$ \\
\hline Flow rate $(\mathrm{m})$ & $17495.71 \mathrm{~kg} / \mathrm{h}$ & $\pm 0.02 \%$ & Gaussian \\
\hline Flow rate $(\mathrm{V})$ & $18000 \mathrm{~L} / \mathrm{h}$ & $\pm 0.25 \%$ & Gaussian \\
\hline Temperature & $80{ }^{\circ} \mathrm{C}$ & $\pm 0.025 \%$ & Gaussian \\
\hline $\begin{array}{c}\text { Absolute } \\
\text { pressure }\end{array}$ & $0.5 \mathrm{MPa}$ & $\pm 0.035 \%$ & Gaussian \\
\hline Collection time & $10 \mathrm{~s}$ & $\pm 1 \cdot 10^{-7}$ & Gaussian \\
\hline
\end{tabular}

The water density has been calculated from IAPWSIF97 formulation, which is associated to a relative uncertainty of $\pm 0,003 \%$ (uniform distribution) compared with experimental data.

The statistical results for the two flow rate measurements, in terms of probability density functions of water mass flow rate, are shown in fig 13. 


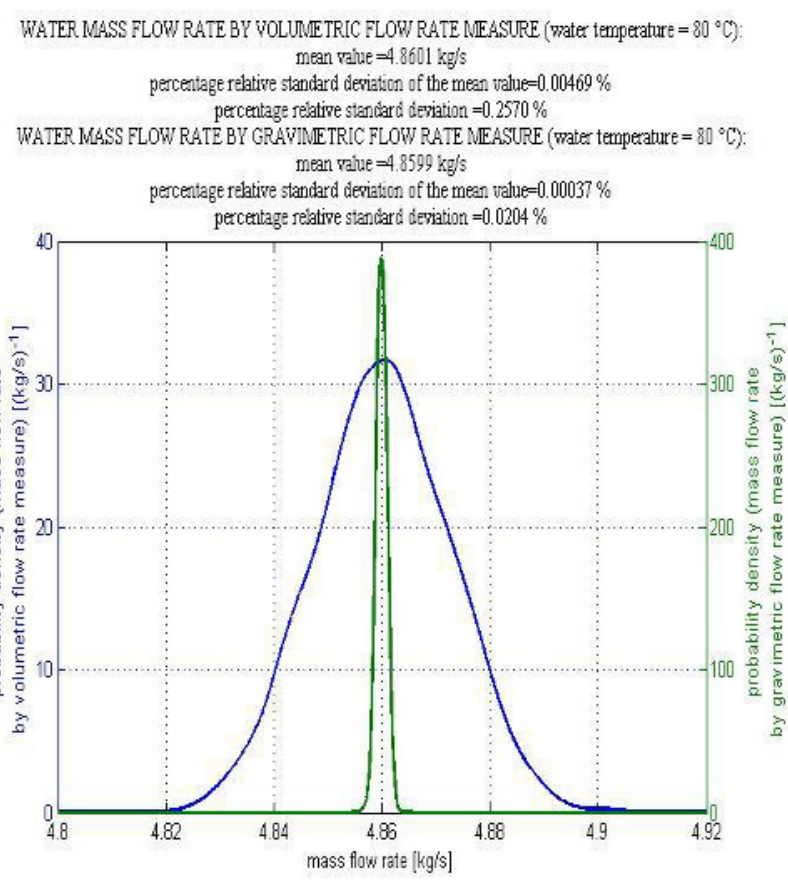

Fig. 13 - Probability density functions for gravimetric and volumetric measurement methods of water flow rate at $80^{\circ} \mathrm{C}$.

The cumulative functions associated with the probability densities, for gravimetric and volumetric measurement methods of water flow rate at $80{ }^{\circ} \mathrm{C}$ rate, are shown in the following figure.

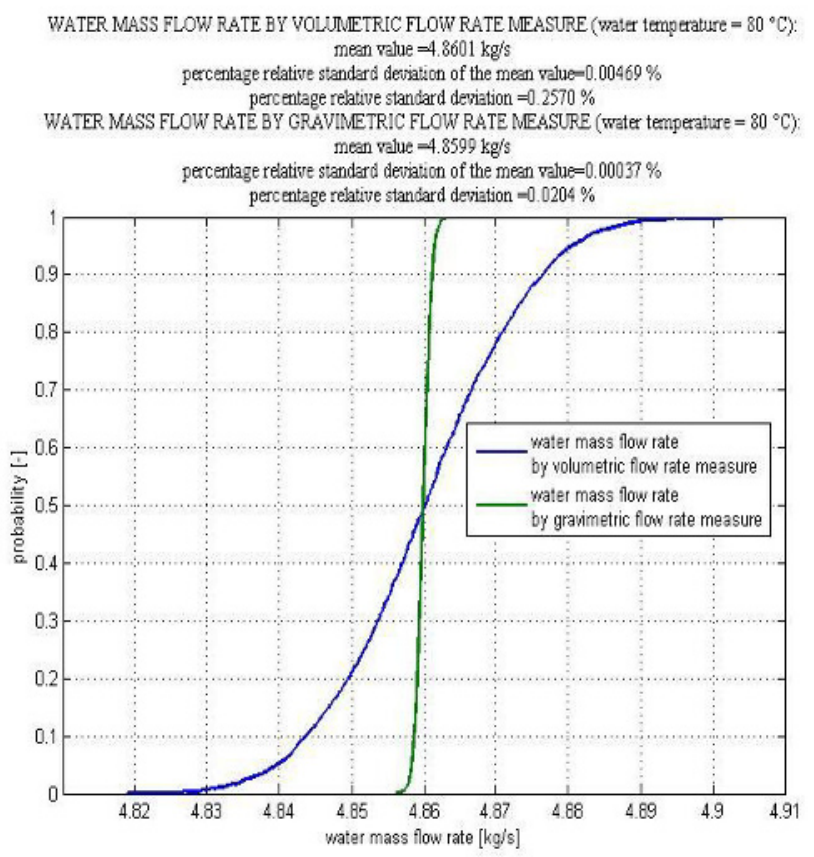

Fig. 14 - Cumulative functions for gravimetric and volumetric measurement methods of water flow rate, at $80{ }^{\circ} \mathrm{C}$.

From the comparison between the percentage relative standard deviations in fig. 13 and in fig 14, we can observe that their main differences are due to the relative uncertainties on volumetric and gravimetric flow rate measuring instruments listed in table $1( \pm 0.25 \%$ for the volumetric flow rate meter and $\pm 0.02 \%$ for the balance).

The Monte Carlo method gives just approximations of the effective probability distributions related to mass flow rate measure, since the number of trials is a finite number. In the estimation of the uncertainty propagation through the calculation procedures used for water mass flow rate measurement, we have to consider the approximation error committed by using a Monte Carlo method, which can be quantified, for instance, by the evaluation of the percentage relative standard deviation of the mean value.

\section{Conclusions}

The renovation and automation of the primary standard for liquid flow measurement at INRIM has allowed a greater reliability in monitoring and measuring the parameters that make up within the field of measurement.

The ability to have a real time a control of the plan setup makes it possible to obtain a more reliable measurement and to reduce the random errors.

The monitoring of the flow diversion, by using of a see-through flow conveyor and a high speed camera, allows a continuous control of the flow jet and the detection of any anomalous behavior that could lead to systematic and hidden errors.

An efficient human-machine interface is realized in order to have a full automated DUT calibration and a more user-friendly and strictly control on all measurement process.

The application of a Monte Carlo method for the estimation of the uncertainty propagation in water flow rate measure has preliminary allowed identifying and selecting the maximum tolerable uncertainties of the measuring instruments, in order to improve the final flow rate standard uncertainty.

\section{References}

1. Rainer Engel, Hans-Joachim Baade, Andreas Rubel "Performance improvement of liquid flow calibrators by applying special measurement and control strategies"$11^{\text {th }}$ Flomeko, may 12-14 2003 - Groningen- NL.

2. Rainer Engel Modeling the uncertainty in liquid flowmeter calibration and application - Requirements and their technical realization for PTB's national water flow standard" - Proceedings Sensor conference-2007

3. IAPWS 2009 Revised Release on the IAPWS Formulation 1995 for the Thermodynamic Properties of Ordinary Water Substance for General and Scientific Use Doorwerth The Netherlands

4. JCGM 101:2008 Evaluation of measurement dataSupplement 1 to the "Guide to the expression of uncertainty in measurement"-Propagation of distributions using a Monte Carlo method" 